\title{
Measurement of Neutrino Oscillations with the MINOS Detectors in the NuMI Beam
}

P. Adamson, ${ }^{9}$ C. Andreopoulos, ${ }^{22}$ K. E. Arms,${ }^{18}$ R. Armstrong, ${ }^{12}$ D. J. Auty, ${ }^{26}$ D. S. Ayres, ${ }^{1}$ B. Baller, ${ }^{9}$ P. D. Barnes, Jr., ${ }^{16}$ G. Barr, ${ }^{20}$ W. L. Barrett, ${ }^{31}$ B. R. Becker, ${ }^{18}$ A. Belias,${ }^{22}$ R. H. Bernstein, ${ }^{9}$ D. Bhattacharya,${ }^{21}$ M. Bishai,${ }^{4}$ A. Blake,${ }^{6}$ G. J. Bock, ${ }^{9}$ J. Boehm, ${ }^{10}$ D. J. Boehnlein, ${ }^{9}$ D. Bogert, ${ }^{9}$ C. Bower, ${ }^{12}$ E. Buckley-Geer, ${ }^{9}$ S. Cavanaugh, ${ }^{10}$ J. D. Chapman, ${ }^{6}$ D. Cherdack, ${ }^{29}$ S. Childress, ${ }^{9}$ B. C. Choudhary, ${ }^{9}$ J. H. Cobb, ${ }^{20}$ S. J. Coleman, ${ }^{32}$ A. J. Culling, ${ }^{6}$ J. K. de Jong, ${ }^{11}$ M. Dierckxsens, ${ }^{4}$ M. V. Diwan, ${ }^{4}$ M. Dorman, ${ }^{17,22}$ S. A. Dytman, ${ }^{21}$ C. O. Escobar, ${ }^{7}$ J. J. Evans,${ }^{17,20}$ E. Falk Harris,${ }^{26}$ G. J. Feldman, ${ }^{10}$ M. V. Frohne, ${ }^{3}$ H. R. Gallagher ${ }^{29}$ A. Godley,${ }^{24}$ M. C. Goodman, ${ }^{1}$ P. Gouffon, ${ }^{23}$ R. Gran, ${ }^{19}$ E. W. Grashorn, ${ }^{18}$ N. Grossman, ${ }^{9}$ K. Grzelak,${ }^{30,20}$ A. Habig, ${ }^{19}$ D. Harris, ${ }^{9}$ P. G. Harris, ${ }^{26}$ J. Hartnell, ${ }^{26,22}$ R. Hatcher, ${ }^{9}$ K. Heller, ${ }^{18}$ A. Himmel,${ }^{5}$ A. Holin, ${ }^{17}$ J. Hylen, ${ }^{9}$ G. M. Irwin, ${ }^{25}$ M. Ishitsuka, ${ }^{12}$ D. E. Jaffe, ${ }^{4}$ C. James, ${ }^{9}$ D. Jensen, ${ }^{9}$ T. Kafka, ${ }^{29}$ S. M. S. Kasahara, ${ }^{18}$ J. J. Kim, ${ }^{24}$ M. S. Kim, ${ }^{21}$ G. Koizumi, ${ }^{9}$ S. Kopp,${ }^{28}$ M. Kordosky, ${ }^{32,17}$ D. J. Koskinen, ${ }^{17}$ S. K. Kotelnikov, ${ }^{15}$ A. Kreymer, ${ }^{9}$ S. Kumaratunga, ${ }^{18}$ K. Lang, ${ }^{28}$ J. Ling, ${ }^{24}$ P. J. Litchfield, ${ }^{18}$ R. P. Litchfield, ${ }^{20}$ L. Loiacono, ${ }^{28}$ P. Lucas, ${ }^{9}$ J. Ma, ${ }^{28}$ W. A. Mann, ${ }^{29}$ A. Marchionni, ${ }^{9}$ M. L. Marshak, ${ }^{18}$ J. S. Marshall, ${ }^{6}$ N. Mayer,${ }^{12}$ A. M. McGowan, ${ }^{1,18}$ J. R. Meier, ${ }^{18}$ G. I. Merzon, ${ }^{15}$ M. D. Messier, ${ }^{12}$ C. J. Metelko, ${ }^{22}$ D. G. Michael, ${ }^{5, *}$ J. L. Miller, ${ }^{14, *}$ W. H. Miller, ${ }^{18}$ S. R. Mishra,${ }^{24}$ C. D. Moore, ${ }^{9}$ J. Morfín, ${ }^{9}$ L. Mualem, ${ }^{5}$ S. Mufson, ${ }^{12}$ S. Murgia, ${ }^{25}$ J. Musser, ${ }^{12}$ D. Naples,${ }^{21}$ J. K. Nelson, ${ }^{32}$ H. B. Newman, ${ }^{5}$ R. J. Nichol, ${ }^{17}$ T. C. Nicholls, ${ }^{22}$ J.P. Ochoa-Ricoux,${ }^{5}$ W. P. Oliver, ${ }^{29}$ R. Ospanov, ${ }^{28}$ J. Paley, ${ }^{12}$ V. Paolone, ${ }^{21}$ A. Para, ${ }^{9}$ T. Patzak,${ }^{8}$ Ž. Pavlović, ${ }^{28}$ G. Pawloski ${ }^{25}$ G. F. Pearce, ${ }^{22}$ C. W. Peck, ${ }^{5}$ E. A. Peterson, ${ }^{18}$ D. A. Petyt,${ }^{18}$ R. Pittam, ${ }^{20}$ R. K. Plunkett, ${ }^{9}$ A. Rahaman, ${ }^{24}$ R. A. Rameika, ${ }^{9}$ T. M. Raufer, ${ }^{22}$ B. Rebel, ${ }^{9}$ J. Reichenbacher, ${ }^{1}$ P. A. Rodrigues, ${ }^{20}$ C. Rosenfeld, ${ }^{24}$ H. A. Rubin, ${ }^{11}$ K. Ruddick, ${ }^{18}$ V. A. Ryabov, ${ }^{15}$ M. C. Sanchez, ${ }^{1,10}$ N. Saoulidou, ${ }^{9}$ J. Schneps, ${ }^{29}$ P. Schreiner, ${ }^{3}$ S.-M. Seun, ${ }^{10}$ P. Shanahan, ${ }^{9}$ W. Smart, ${ }^{9}$ C. Smith,${ }^{17}$ A. Sousa, ${ }^{20}$ B. Speakman, ${ }^{18}$ P. Stamoulis, ${ }^{2}$ M. Strait, ${ }^{18}$ P. Symes,${ }^{26}$ N. Tagg, ${ }^{29}$ R. L. Talaga, ${ }^{1}$ M. A. Tavera, ${ }^{26}$ J. Thomas, ${ }^{17}$ J. Thompson, ${ }^{21, *}$ M. A. Thomson, ${ }^{6}$ J. L. Thron, ${ }^{1}$ G. Tinti, ${ }^{20}$ I. Trostin, ${ }^{13}$ V. A. Tsarev,${ }^{15}$ G. Tzanakos, ${ }^{2}$ J. Urheim,,${ }^{12}$ P. Vahle,${ }^{32,17}$ B. Viren, ${ }^{4}$ C. P. Ward, ${ }^{6}$ D. R. Ward, ${ }^{6}$ M. Watabe, ${ }^{27}$ A. Weber, ${ }^{20}$ R. C. Webb,${ }^{27}$ A. Wehmann, ${ }^{9}$ N. West,${ }^{20}$ C. White, ${ }^{11}$ S. G. Wojcicki, ${ }^{25}$ D. M. Wright, ${ }^{16}$ T. Yang, ${ }^{25}$ M. Zois, ${ }^{2}$ K. Zhang, ${ }^{4}$ and R. Zwaska ${ }^{9}$

(MINOS Collaboration)

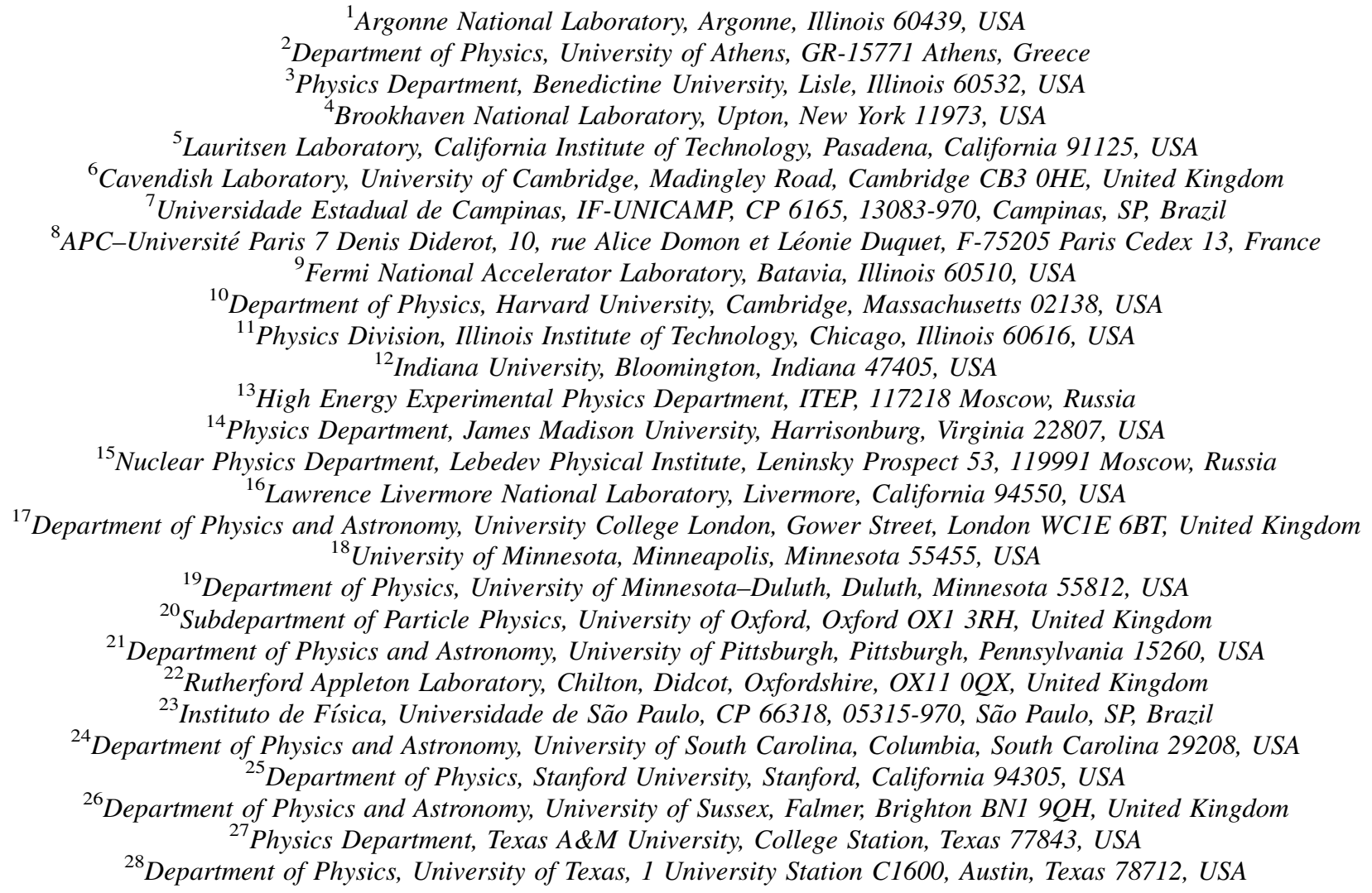


${ }^{29}$ Physics Department, Tufts University, Medford, Massachusetts 02155, USA

${ }^{30}$ Department of Physics, Warsaw University, PL-00-681 Warsaw, Poland

${ }^{31}$ Physics Department, Western Washington University, Bellingham, Washington 98225, USA

${ }^{32}$ Department of Physics, College of William \& Mary, Williamsburg, Virginia 23187, USA

(Received 13 June 2008; published 26 September 2008)

This Letter reports new results from the MINOS experiment based on a two-year exposure to muon neutrinos from the Fermilab NuMI beam. Our data are consistent with quantum-mechanical oscillations of neutrino flavor with mass splitting $\left|\Delta m^{2}\right|=(2.43 \pm 0.13) \times 10^{-3} \mathrm{eV}^{2}(68 \%$ C.L. $)$ and mixing angle $\sin ^{2}(2 \theta)>0.90$ (90\% C.L.). Our data disfavor two alternative explanations for the disappearance of neutrinos in flight: namely, neutrino decays into lighter particles and quantum decoherence of neutrinos, at the 3.7 and 5.7 standard-deviation levels, respectively.

DOI: 10.1103/PhysRevLett.101.131802

Several experiments [1-8] have produced compelling evidence of the disappearance of neutrinos of a given lepton flavor. In previous publications [8], the MINOS experiment has also presented evidence for energydependent disappearance of muon neutrinos produced by the NuMI facility at Fermilab. Based on the number of events, that result provides evidence of the disappearance of $\nu_{\mu}$ at a level of 5.2 standard deviations. Such observations support the description of neutrinos via two independent basis sets, the mass and the flavor eigenstates, with the bases related by the $3 \times 3$ Pontecorvo-Maki-NakagawaSakata (PMNS) matrix [9]. They imply that at least two of the neutrino eigenstates have nonzero mass. In this Letter we present results obtained from a larger data set than that used in [8]. These results provide a precision measurement of the oscillation parameters and furthermore disfavor two other theoretical interpretations of neutrino flavor disappearance $[10,11]$.

The MINOS detectors [12] and the NuMI beam line [13] are described elsewhere. In brief, NuMI is a conventional two-horn-focused neutrino beam with a $675 \mathrm{~m}$ long decay tunnel. The horn current and position of the hadron production target relative to the horns can be configured to produce different $\nu_{\mu}$ energy spectra. MINOS consists of two detectors: a $0.98 \mathrm{kt}$ near detector (ND) $1.04 \mathrm{~km}$ from the NuMI target, and a $5.4 \mathrm{kt}$ far detector (FD) $735 \mathrm{~km}$ from the target. Both are segmented, magnetized calorimeters that permit particle tracking. The curvature of muons produced in $\nu_{\mu}+\mathrm{Fe} \rightarrow \mu^{-}+X$ interactions [14] is used for energy determination of muons that exit the detector and to distinguish the $\nu_{\mu}$ component of the beam from the $6 \%$ intrinsic $\bar{\nu}_{\mu}$ contamination. The energy of muons contained in the detector is measured via their range. Oscillations of $\nu_{\mu}$ into other neutrino flavors result in an energydependent depletion of $\nu_{\mu}$ interactions in the FD relative to the expectation based upon the ND measurement.

This Letter describes results from data recorded between May 2005 and July 2007. Over this period, a total of $3.36 \times$ $10^{20}$ protons on target (POT) were accumulated for this analysis. A $1.27 \times 10^{20}$ POT subset of this exposure (hereafter referred to as run I) forms the data set from Ref. [8]. In
PACS numbers: 14.60.Lm, 14.60.Pq, 29.27.-a, 29.30.-h

run I and for most of the new running period (run II), the beam line was configured to enhance $\nu_{\mu}$ production with energies $1-5 \mathrm{GeV}$ (the low-energy configuration). An exposure of $0.15 \times 10^{20} \mathrm{POT}$ was accumulated with the beam line configured to enhance the $\nu_{\mu}$ energy spectrum at 5-15 GeV (the high-energy configuration). The run II data were collected with a replacement target of identical construction due to failure of the motion system of the first target. The new target was found to be displaced longitudinally $\sim 1 \mathrm{~cm}$ relative to the first target, resulting in a $30 \mathrm{MeV}$ shift in the neutrino spectrum. This effect is incorporated in the Monte Carlo simulation, and the run I and run II data sets are analyzed separately to account for this shift.

The simulation of neutrino production and detection is accomplished with a model of hadron production in the

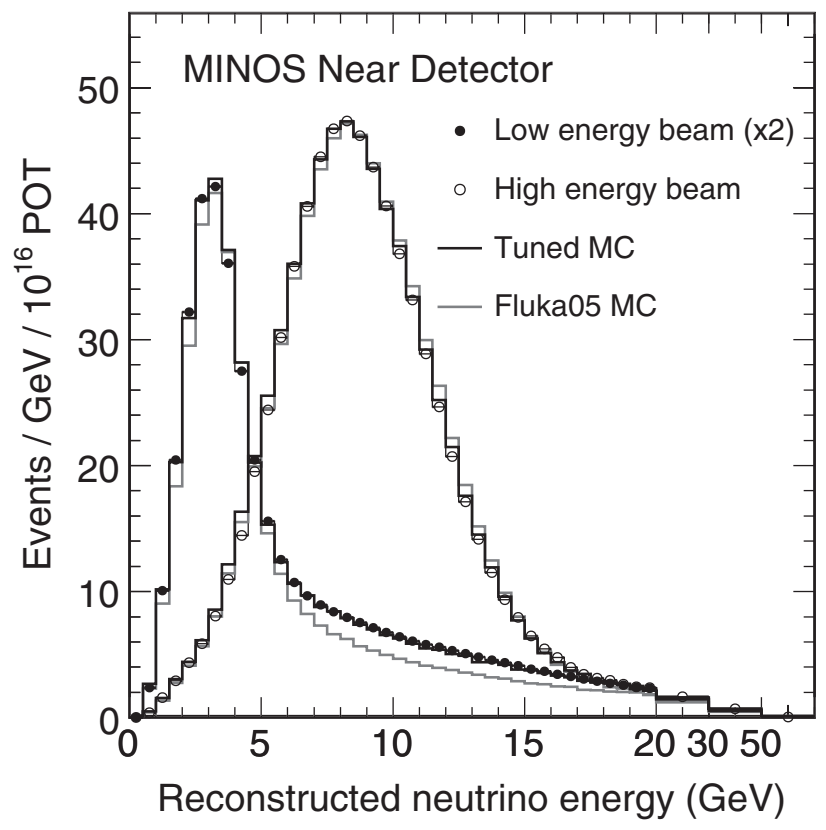

FIG. 1. Energy spectra in the MINOS ND for two of the nine beam configurations before and after tuning the Monte Carlo simulation to the ND data. The data combine run I and run II. Both configurations are utilized in the oscillation analysis. 
target using FLUKA [15] and a GEANT3 [16] simulation of the beam line and detector. NEUGEN3.5.5 [17], tuned to data from previous bubble chamber neutrino experiments and experiments with pion beams scattering on iron, is used to model neutrino interactions. As in our previous analysis, the Monte Carlo (MC) simulation of the neutrino flux was constrained to agree with the neutrino energy spectrum in the ND collected in nine different configurations of the NuMI beam [8], thereby reducing uncertainties in the flux prediction at the FD. Figure 1 compares the simulation to the ND data acquired in the two configurations used in the oscillation analysis.

Neutrino interactions in the MINOS detectors can either be charged current, $\nu_{\mu}+\mathrm{Fe} \rightarrow \mu^{-}+X$, or neutral current, $\nu_{\mu}+\mathrm{Fe} \rightarrow \nu_{\mu}+X$. In this analysis, only the former are used because they identify the interacting neutrino flavor and because the reconstructed energy best measures the full neutrino energy. To select charged-current events, we have implemented a new algorithm [18] based on a multivariate likelihood including four variables that characterize a muon track: the event length, the average pulse height per plane along the track, the transverse energy deposition profile of the track, and the fluctuation of the energy deposited in scintillator strips along the track. The new selection algorithm, along with a new track-finding algorithm, improves our efficiency to identify and select charged-current interactions in the FD from $75.3 \%$ using the previous selection [8] to $81.5 \%$ in the current selection, in the absence of oscillations. The new selection reduces

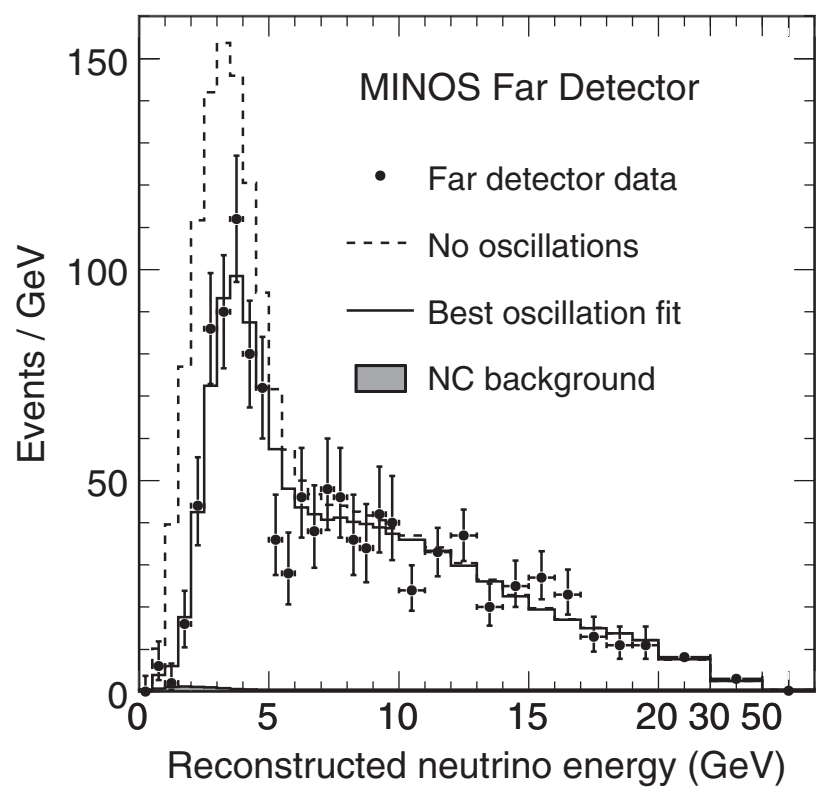

FIG. 2. Comparison of the FD data (points, with statistical uncertainties) from the low- and high-energy configurations with the predictions for the $\nu_{\mu}$ energy spectra with and without the effect of oscillations. The estimated neutral-current (NC) background is indicated. the neutral-current contamination in the charged-current sample from $1.8 \%$ in our previous publication to $0.6 \%$ in the present analysis. The present analysis uses a larger fiducial mass of $4.17 \mathrm{kt}$ in the FD, an increase of $2.9 \%$ over the mass used in [8].

The measured energy spectrum at the ND is used to predict the energy spectrum at the FD. As in our previous analysis [8], we compute a transfer matrix to correct for $\sim 20 \%$ differences expected in the shape of the energy spectrum in the FD relative to the ND that arise from meson decay kinematics and from beam line geometry $[8,19]$. We have cross-checked this technique by comparison to other calculations of the FD spectrum [8].

The FD energy spectra were inspected only after the analysis procedure was finalized and basic data integrity checks were performed. We observe 848 events in the FD for all energies $0-120 \mathrm{GeV}$ produced by the NuMI beam, compared to the unoscillated expectation of $1065 \pm$ 60 (syst). In the low-energy configuration alone, the number of events observed in the data is 730 , to be compared with an expectation of $936 \pm 53$ (syst). The observed energy spectrum of the events from the low- and high-energy data sets is shown along with the predicted spectrum in Fig. 2, and the ratio of these data to the expected spectrum is shown in Fig. 3.

Under the assumption the observed deficit is due to $\nu_{\mu} \rightarrow \nu_{\tau}$ oscillations [20], a fit is performed to extract the parameters $\left|\Delta m^{2}\right|$ and $\sin ^{2}(2 \theta)$ [21] using the expression

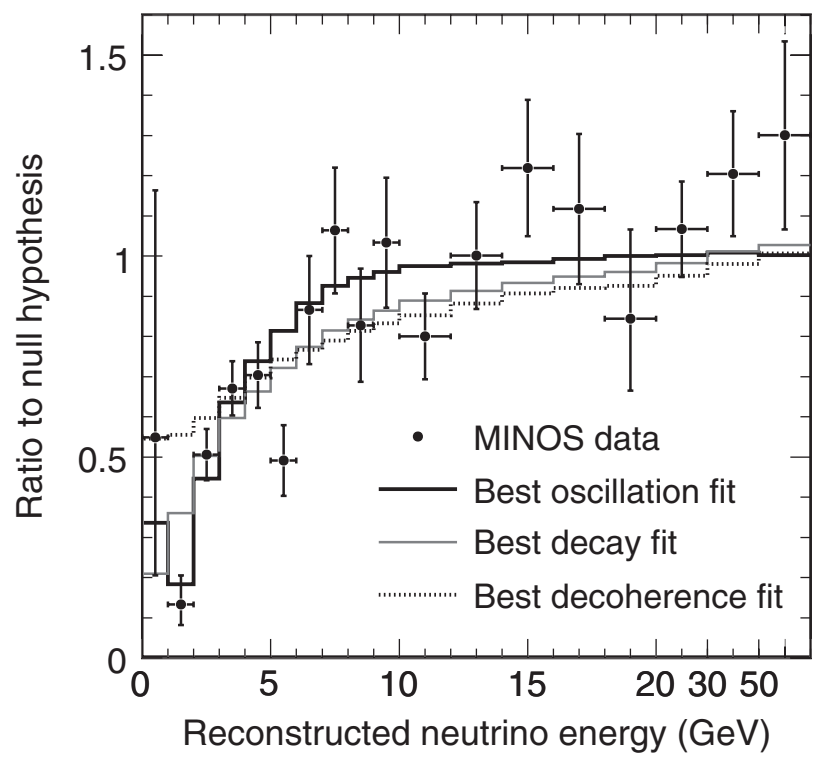

FIG. 3. Ratio of the FD data and the expected spectrum in the absence of oscillations. Also shown are the best-fit curve to Eq. (1) and the best fit to alternative models of neutrino disappearance $[10,11]$. For display purposes, the data have been rebinned and the estimated oscillated NC background is subtracted. 


$$
P\left(\nu_{\mu} \rightarrow \nu_{\mu}\right)=1-\sin ^{2}(2 \theta) \sin ^{2}\left(1.27 \Delta m^{2} \frac{L}{E}\right)
$$

where $L[\mathrm{~km}]$ is the distance from the target, $E[\mathrm{GeV}]$ is the neutrino energy, and $\left|\Delta m^{2}\right|$ is measured in $\mathrm{eV}^{2}$. The separate FD spectra from run I, run II, and the highenergy run, binned as in Fig. 2, are simultaneously fit to Eq. (1). The best-fit parameters minimize the $\chi^{2}$ expression given in [8]. The predicted oscillated spectrum includes the contamination from $\nu_{\tau}$ produced in the oscillation process.

The effects of systematic uncertainties were evaluated by fitting modified MC simulations in place of data. Table I gives the differences between the fitted values obtained with the modified and an unmodified MC simulation. The largest effects are (a) the $\pm 10.3 \%$ uncertainty in the absolute hadronic energy scale, which is the sum in quadrature of a $\pm 5.7 \%$ error in the calorimeter response to hadrons as derived from test beam measurements [22], a $\pm 2.3 \%$ uncertainty in the energy scale calibration, and a $\pm 8.2 \%$ uncertainty in the simulation of neutrino production of hadrons in iron nuclei; (b) the $\pm 3.3 \%$ relative uncertainty in the hadronic energy scale between the ND and FD; (c) the $\pm 4.0 \%$ uncertainty on the predicted FD event rate which is the sum in quadrature of the uncertainties on the detectors' fiducial mass, event selection efficiency, and the POT counting; (d) the $\pm 50 \%$ uncertainty on the neutralcurrent contamination in the charged-current event sample; and (e) the uncertainty on the muon momenta measured via range $( \pm 2.0 \%)$ or curvature $( \pm 3.0 \%)$.

In fitting the data to Eq. (1), $\sin ^{2}(2 \theta)$ was constrained to lie in the physical region. To reduce the effect of the dominant systematic uncertainties in Table I [(a) and (c) for $\left|\Delta m^{2}\right|$, (d) for $\left.\sin ^{2}(2 \theta)\right]$ these three systematic uncertainties were included as nuisance parameters in the fit. The resulting best fit to the neutrino energy spectrum is shown in Fig. 2 and 3. We obtain $\left|\Delta m^{2}\right|=(2.43 \pm 0.13) \times$ $10^{-3} \mathrm{eV}^{2}$ and $\sin ^{2}(2 \theta)>0.95$ at $68 \%$ C.L. [23]. The fit $\chi^{2}=90$ for 97 degrees of freedom. The resulting $68 \%$ C.L. $\left(\Delta \chi^{2}=2.30\right)$ and $90 \%$ C.L. $\left(\Delta \chi^{2}=4.61\right)$ intervals for the oscillation parameters $\left|\Delta m^{2}\right|$ and $\sin ^{2}(2 \theta)$ are shown in Fig. 4 [24]. The MC simulation predicts negligible backgrounds of 0.7 events from cosmic ray muons, and, at the best-fit value for $\left|\Delta m^{2}\right|$ and $\sin ^{2}(2 \theta), 2.3$ events from neutrino interactions in the upstream rock, 5.9 neutral current and $1.5 \nu_{\tau}$ events in the final sample. If the fit is not constrained to the physical region, $\left|\Delta m^{2}\right|=2.33 \times$ $10^{-3} \mathrm{eV}^{2}$ and $\sin ^{2}(2 \theta)=1.07$, with a 0.6 unit decrease in $\chi^{2}$. Correspondingly, the contours in Fig. 4 are smaller than those expected for the present data set. Our measurement is the most precise determination of the mass splitting $\left|\Delta m^{2}\right|$.

Figure 4 also shows that the previous MINOS result [8] is in good agreement with the current measurement. Taken alone, the run II data yield $\left|\Delta m^{2}\right|=\left(2.32_{-0.16}^{+0.17}\right) \times$ $10^{-3} \mathrm{eV}^{2}$ and $\sin ^{2}(2 \theta)=1.0$, to be compared with $\left(2.57_{-0.20}^{+0.23}\right) \times 10^{-3} \mathrm{eV}^{2}$ and $\sin ^{2}(2 \theta)=1.0$ from run $\mathrm{I}$. The two results are consistent at $68 \%$ C.L. We note that the value of $2.57 \times 10^{-3} \mathrm{eV}^{2}$ for run I differs from that quoted in [8] because of our improved reconstruction and selection of charged-current events and improved MC simulation of neutrino interactions.

We have also fit the FD energy spectra to alternative models that have been proposed to explain the disappearance of neutrinos in flight, namely, the decay of neutrinos to lighter particles [Eq. 13 of [10]], and the decoherence of the neutrino's quantum-mechanical wave packet [Eq. 5 of [11]]. Figure 3 shows the ratios of the energy spectra arising from our best fits to these alternative models to the prediction of the FD spectrum in the absence of $\nu_{\mu}$ disappearance. The $\chi^{2}$ for the best fit to the decay model is $104 / 97$ d.o.f., while that for the decoherence model is

TABLE I. Sources of systematic uncertainties in the measurement of $\left|\Delta m^{2}\right|$ and $\sin ^{2}(2 \theta)$. The values are the average shifts for varying the parameters in both directions without imposing the $\sin ^{2}(2 \theta) \leq 1$ constraint on the fit. The shift resulting from each systematic effect is evaluated individually. The dominant uncertainties are incorporated as nuisance parameters in the fit of our data to Eq. (1) so as to reduce their effect on the oscillation parameter measurement (see text).

\begin{tabular}{lcc}
\hline \hline \multicolumn{1}{c}{ Uncertainty } & $\begin{array}{c}\left|\Delta m^{2}\right| \\
\left(10^{-3} \mathrm{eV}^{2}\right)\end{array}$ & $\sin ^{2}(2 \theta)$ \\
\hline (a) Absolute hadronic $E$ scale $( \pm 10.3 \%)$ & 0.052 & 0.004 \\
(b) Relative hadronic $E$ scale $( \pm 3.3 \%)$ & 0.027 & 0.006 \\
(c) Normalization $( \pm 4 \%)$ & 0.081 & 0.001 \\
(d) NC contamination $( \pm 50 \%)$ & 0.021 & 0.016 \\
(e) $\mu$ momentum $($ range $2 \%$, curvature $3 \%)$ & 0.032 & 0.003 \\
(f) $\sigma_{\nu}\left(E_{\nu}<10 \mathrm{GeV}\right)( \pm 12 \%)$ & 0.006 & 0.004 \\
(g) Beam flux & 0.010 & 0.000 \\
Total systematic uncertainty & 0.108 & 0.018 \\
Expected statistical uncertainty & 0.19 & 0.09 \\
\hline \hline
\end{tabular}




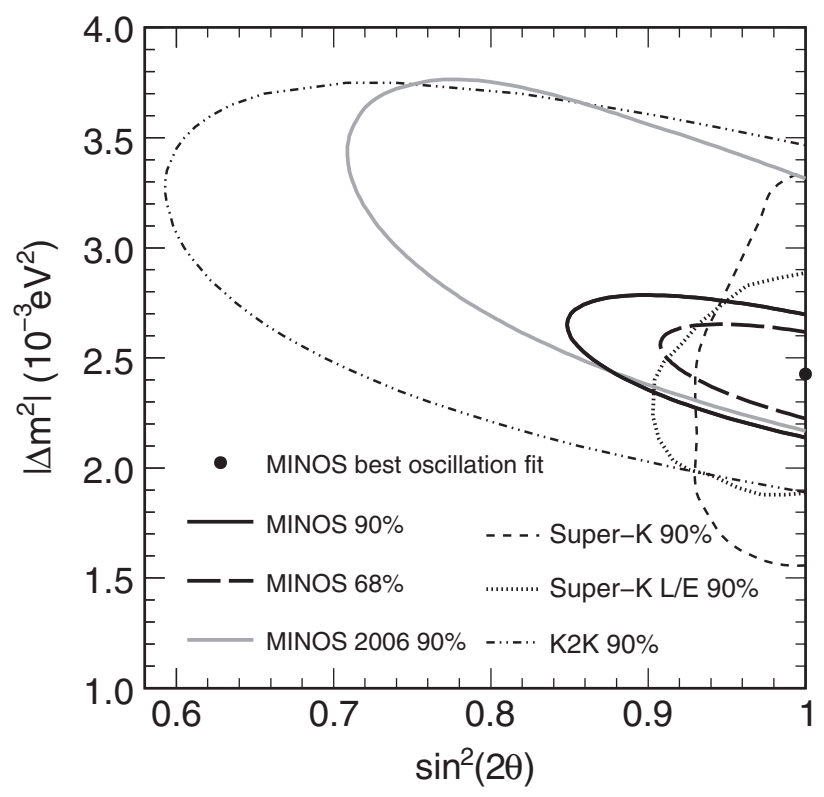

FIG. 4. Contours for the oscillation fit to the data in Fig. 2, including systematic errors. Also shown are contours from previous experiments $[1,4]$ and our earlier result [8].

123/97 d.o.f.. Given the $\Delta \chi^{2}=14$ and 33 of these two models relative to the oscillation hypothesis, these models are disfavored with respect to the oscillation hypothesis at the 3.7 and 5.7 standard-deviation levels.

In summary, we have presented updated measurements of neutrino oscillation parameters from the MINOS experiment. Based upon an exposure of $3.36 \times 10^{20}$ POT from the NuMI beam, we obtain $\left|\Delta m^{2}\right|=(2.43 \pm 0.13) \times$ $10^{-3} \mathrm{eV}^{2}\left(68 \%\right.$ C.L.) and mixing angle $\sin ^{2}(2 \theta)>0.90$ (90\% C.L.). As the data set presented here includes the subset analyzed in [8], these results supersede our previous publication. Our data disfavor two alternative explanations for disappearance of neutrinos in flight: namely, neutrino decays [10] into lighter particles and quantum decoherence of neutrinos [11] at the 3.7 and 5.7 standard-deviation level, respectively.

This work was supported by the U.S. DOE, the U.K. STFC, the U.S. NSF, the State and University of Minnesota, the University of Athens, Greece, and Brazil's FAPESP and CNPq. We are grateful to the Minnesota Department of Natural Resources, the crew of the Soudan Underground Laboratory, and the staff of Fermilab for their contribution to this effort.

*Deceased.

[1] Y. Ashie et al., Phys. Rev. Lett. 93, 101801 (2004); Phys. Rev. D 71, 112005 (2005).

[2] W. W. M. Allison et al., Phys. Rev. D 72, 052005 (2005).

[3] M. Ambrosio et al., Eur. Phys. J. C 36, 323 (2004).

[4] M. H. Ahn et al., Phys. Rev. D 74, 072003 (2006).

[5] J. Hosaka et al., Phys. Rev. D 73, 112001 (2006).

[6] S. N. Ahmed et al., Phys. Rev. Lett. 92, 181301 (2004).

[7] T. Araki et al., Phys. Rev. Lett. 94, 081801 (2005).

[8] D. G. Michael et al., Phys. Rev. Lett. 97, 191801 (2006); P. Adamson et al., Phys. Rev. D 77, 072002 (2008).

[9] B. Pontecorvo, JETP 34, 172 (1958); Z. Maki, M. Nakagawa, and S. Sakata, Prog. Theor. Phys. 28, 870 (1962).

[10] V. Barger et al., Phys. Rev. Lett. 82, 2640 (1999).

[11] G. L. Fogli et al., Phys. Rev. D 67, 093006 (2003).

[12] D. G. Michael et al. (to be published).

[13] S. Kopp, arXiv:physics/0508001.

[14] Approximately 5\% of $\nu_{\mu}$ interactions occur in aluminum and scintillator.

[15] A. Fasso et al., CERN Report No. CERN-2005-10, INFN Report No. INFN/TC_05/11, SLAC Report No. SLAC-R773, 2005.

[16] R. Brun et al., CERN Program Library Report No. W5013, 1984.

[17] H. Gallagher, Nucl. Phys. B, Proc. Suppl. 112, 188 (2002); update available at arXiv:0806.2119.

[18] R. Ospanov, Ph.D. Thesis, UT-Austin, 2008.

[19] M. Szleper and A. Para, arXiv:hep-ex/0110001.

[20] W.-M. Yao et al., J. Phys. G 33, 1 (2006).

[21] The experiment measures an unresolved mixture of $\left|\Delta m_{31}^{2}\right|$ and $\left|\Delta m_{32}^{2}\right|$, which we refer to as $\left|\Delta m^{2}\right|$ for brevity. The parameter $\sin ^{2}(2 \theta)$ is likewise an admixture. For further discussion, see G.L. Fogli et al., Prog. Part. Nucl. Phys. 57, 742 (2006).

[22] P. Adamson et al., Nucl. Instrum. Methods Phys. Res., Sect. A 556, 119 (2006).

[23] Although the contours in Fig. 4 are calculated with 2 d.o.f, the parameter errors are calculated with only 1 d.o.f. as in [20] using $\Delta \chi^{2}=1$ and 2.71, respectively.

[24] The effect of the constraint to the physical region was investigated using the unified approach of G. J. Feldman and R. D. Cousins, Phys. Rev. D 57, 3873 (1998), which gave slightly smaller confidence intervals. 